\title{
Reconstructing the observation process to correct for changing detection probability of a critically endangered species
}

\author{
A. J. McConville', Iu. A. Grachev ${ }^{2}$, A. Keane ${ }^{1,3}$, T. Coulson ${ }^{3}$, A. B. Bekenov ${ }^{2}$, \\ E. J. Milner-Gulland ${ }^{1,3, *}$ \\ ${ }^{1}$ Centre for Environmental Policy, Imperial College London, Exhibition Road, London SW7 2AZ, UK \\ ${ }^{2}$ Laboratory of Theriology, Institute of Zoology, Ministry of Education and Science, Akademgorodok, \\ Almaty 480032, Kazakhstan \\ ${ }^{3}$ Department of Life Sciences and Centre for Population Biology, Imperial College London, Silwood Park Campus, \\ Buckhurst Road, Ascot, Berkshire SL5 7PY, UK
}

\begin{abstract}
Effective conservation decision-making requires robust estimates of population trends. It is often assumed that, as long as monitoring methods remain consistent over time, trends in relative abundance are valid proxies for actual abundance. However, if the bias and uncertainty of relative abundance estimates change over time, this can have a serious impact on the validity of monitoring programmes. We developed a simple model for the retrospective assessment of the likely error and bias in abundance estimates from aerial surveys of the saiga antelope. Due to dramatic reductions in group size and density, current estimates of abundance are probably substantially lower than the true population size, and the level of uncertainty surrounding these estimates precludes their use for monitoring trends. This has implications for the Government of Kazakhstan's ability to monitor progress towards their agreed conservation goals. The method is potentially widely applicable to species for which historical data on relative abundance and group size are available.
\end{abstract}

KEY WORDS: Monitoring · Saiga tatarica $\cdot$ Antelope · Aerial survey $\cdot$ Kazakhstan · Distance · Population trend $\cdot$ Ungulate $\cdot$ Relative abundance

\section{INTRODUCTION}

A fundamental objective of monitoring the status of populations is the identification of trends in their size. This is often challenging because constraints in funding, logistics and capacity determine the type of methods that can be employed. Hence, in many cases, the methods used are known to be unsuitable for estimation of absolute abundance; instead, the results are presented as trends in relative abundance (e.g. many bird surveys; Gregory et al. 2004). A key assumption when using relative abundance trends for wildlife monitoring is that detection probability is constant over time and space (Pollock et al. 2002). This assumption may be adequate when survey methods are applied consistently and the population is relatively stable (e.g. Schauster et al. 2002). However, many species of conservation concern are not only declining rapidly, but are undergoing changes in their population biology which are likely to influence detectability (e.g. changes in group size linked to Allee effects; Stephens \& Sutherland 1999). Examples of situations in which changes in detectability are known to be an issue are long-term bird surveys and fisheries. For birds, Norvell et al. (2003) found that relative abundance estimates were biased and unreliable as compared to distance sampling methods over a 7-yr period, while Hochachka \& Fiedler (2008) showed that changes in bird behaviour confounded trends obtained from long-term migration ringing studies. In fisheries, trends in rela- 
tive abundance are often obtained from catch per unit effort (CPUE) data. However, the use of these data relies on strict assumptions about the relationship between catch and effort, which is affected by fish responses to exploitation, both behavioural and demographic (Walters 2003). There is increasing empirical evidence to suggest that these responses lead to nonlinear relationships between CPUE and abundance (e.g. Lorenzen et al. 2006). If the bias and uncertainty of relative abundance estimates change over time, this can have a serious impact on the validity of monitoring programmes (Pollock et al. 2002), and hence on our ability to plan conservation interventions and assess their effectiveness. Our ability to quantify these biases is often limited by lack of data and nonexistent validation of the methods employed.

We examined this issue for the saiga antelope Saiga tatarica. This nomadic species of the Eurasian steppes and semi-deserts was listed as Critically Endangered in the International Union for Conservation of Nature (IUCN) Red Lists in 2001 on the basis of a >95\% decline in population size over a 10-yr period (MilnerGulland et al. 2001; www.redlist.org). In 2006, the range states signed a Memorandum of Understanding (MOU) under the Convention on Migratory Species (CMS) with the medium-term goal of stabilisation and, where possible, an increase in saiga populations over a 5 -yr period; this goal is to be assessed by estimates of population size made using appropriate methods, with an accompanying assessment of uncertainty (CMS 2006a). Current monitoring methods provide an annual population estimate with no measure of uncertainty. Given the major population crash experienced by the saiga, it is likely that density, group size and distribution of the population have all changed dramatically over the last 10 to $15 \mathrm{yr}$. Therefore, although the massive overall decline is not in question, it is doubtful that any recently reported abundance trends are reliable. This means that it may not be possible to assess progress made by range states towards their agreed goal. We used data on observed group sizes from aerial surveys to reconstruct the observation process. This allowed us to obtain both an estimate and range for the true population size and an understanding of the effect of underlying biological changes on observation bias. Our simple procedure is likely to be widely applicable to other species for which historical trends in relative abundance are available.

\section{MATERIALS AND METHODS}

In Kazakhstan, the saiga has been monitored by aerial survey, using consistent methods over the last several decades (Grachev 2004). The survey team flies transects over the areas of highest saiga density, as determined from local and expert knowledge. Transects are, where possible, $10 \mathrm{~km}$ apart and the assumed strip width is $1 \mathrm{~km}$ each side of the aircraft, such that $20 \%$ of the area is covered. The team then extrapolates a population estimate by dividing the number of saigas seen by the coverage (usually 0.2 ) and multiplying by the size of the area of saiga concentration, calculated by drawing a line around the observations and counting grid squares within that line. There is no measure of error. The observations consist of the estimated size and approximate location of each group seen. The surveys are carried out in April, when the saigas still have pale coats but the snow has gone, and when they typically aggregate in relatively large migratory groups (Bekenov et al. 1998). This survey technique was developed during Soviet times, and thus does not follow standard Western protocols (e.g. Norton-Griffiths 1978, Buckland et al. 2001). We obtained data from 2 such aerial surveys for 1 saiga population (the Ustiurt population), undertaken in very different circumstances. In 1998, the population was large, estimated at 246000 , with a density of around 80 animals $\mathrm{km}^{-2}$, and $76 \%$ of the population in herds $\geq 500$ ind. in size ( $3 \%$ were in herds of $\leq 50$ ind.). In 2006, the aerial survey gave a population estimate of 13500 , with a density of $1 \mathrm{~km}^{-2}$, and $66 \%$ of the population in herds $\leq 50$ animals in size (none were $\geq 500$ ).

The potential biases in aerial survey are well understood, and include the effect of distance from the aircraft and animal group size on observability, the effect of variation in aircraft height on the effective strip width, inconsistency in bearings leading to deviation from the transect, and variations in observer performance both in counting groups and estimation of distance (Jolly 1969, Samuel \& Pollock 1981, Caughley \& Grice 1982, Drummer \& McDonald 1987, Samuel et al. 1987, Bayliss \& Yeomans 1989). The issues which we address are firstly, the interaction between group size and distance from the aircraft in determining the detectability of a group, and secondly, the effect of overall density on precision of the estimate. Our expectation is that the fewer groups there are, the more variability there will be in whether a group is on a transect (assuming the area surveyed and coverage remain constant), and the smaller a group is, the less likely it is to be detected, with this detectability declining non-linearly with distance from the aircraft. There are other issues with the Kazakhstan survey which we do not address, including unsystematic coverage of the survey area and poor practice when flying, introducing further, largely unquantifiable, error and bias (Norton-Griffiths \& McConville 2007). Hence, our results are minimum estimates of the levels of uncertainty. 
The datasets give information only on the estimated size of each group observed in the 1998 and 2006 surveys, so our analysis required 2 distinct steps: (1) reconstructing the true population distribution underlying the observed data, and then (2) simulating the observation process in a virtual landscape populated with saiga groups derived from this distribution. The first part of the modelling process was to create a bias-correction algorithm for a given survey year (Fig. 1). We fitted a lognormal distribution to the observed group size distribution in the raw data. The log-normal curves were good fits to the observed group size distribution data both for $1998(\log \mu=4.72 \pm 0.15, \log \sigma=1.54 \pm$ $0.11)$ and $2006(\log \mu=2.90 \pm 0.10, \log \sigma=0.99 \pm 0.07)$ : all data are $\pm \mathrm{SD}_{i} \log \mu$ is the $\log$ of the group size distribution; $\log \sigma$ is the log of the SD of the group size distribution. This observed distribution, however, almost certainly underestimated the proportion of smaller groups in the population. Observers are not able to detect all of the groups that occur within their sampling area, and it is probable that small groups are less conspicuous than larger groups and are therefore missed more often. Samuel \& Pollock (1981) proposed a simple method for estimating the actual distribution of group sizes as a function of the observed distribution:

$$
q=1-s \exp ^{-t g}
$$

where $q$ is the probability that a group is missed, $s$ is the probability that an individual animal is missed, $t$ is the rate at which the function approaches the asymptote and $g$ is the number of individuals within the group. We estimated these parameters to be $s=0.5$ and $t=0.1$, based on expert knowledge (Iu. A. Grachev).

We divided the observed distribution of group sizes by the probability that each group size is detected, $q$, to give a corrected log-normal distribution for saiga group size. We next populated a virtual landscape with a saiga population of a given total size, with group sizes taken from the corrected log-normal distribution and randomly distributed in space. We simulated the observation process by sampling $20 \%$ of this area, repeated 1000 times. The observation process required a function for the probability of detecting a group, dependent on group size and distance. The function used was a reverse logistic (Eq. 2), similar to that estimated for pronghorn Antilocapra americana (Guenzel 1997) and marsh deer Blastocerus dichotomus (Andriolo et al. 2005), which has a pronounced point of inflection where detectability drops off rapidly:

$$
p=1-\frac{\exp (a+b[x-i])}{1+\exp (a+b[x-i])}
$$

where $p$ is the probability of detecting an animal at distance $x, a$ and $b$ are shape parameters, and $i$ is the point of inflection. The value of $i$ was calculated as:

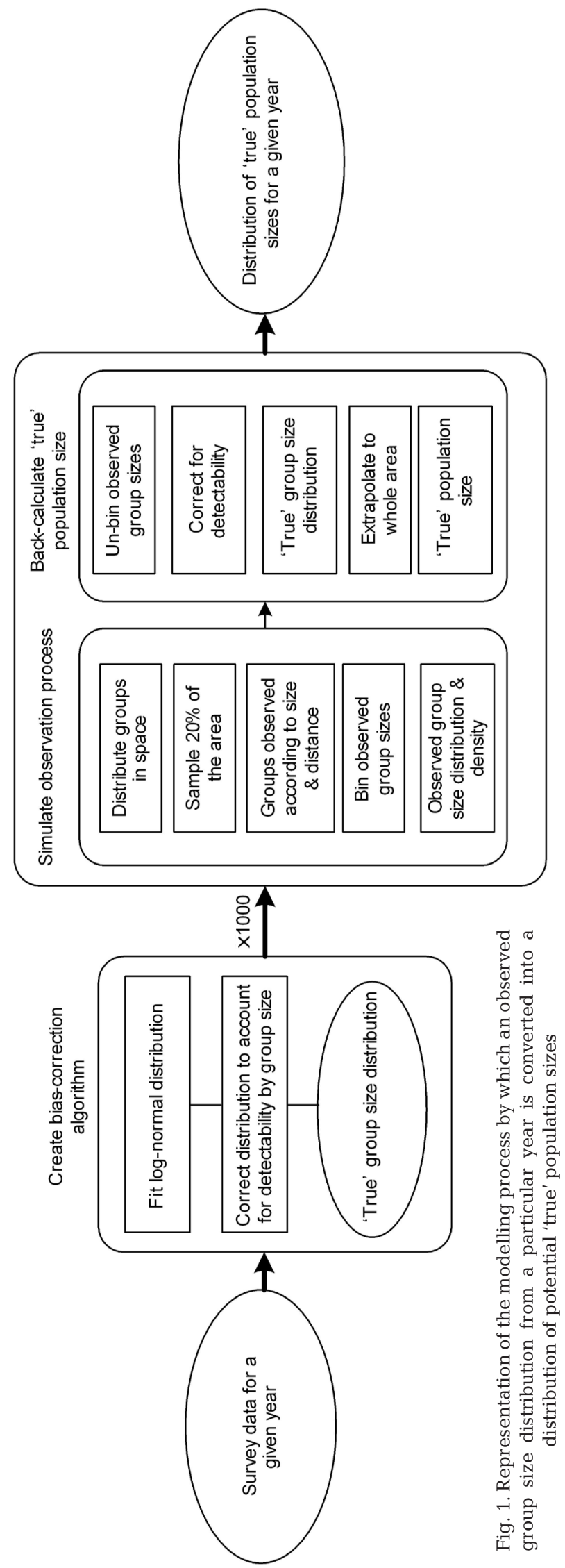




$$
i=m g+\mathrm{C}
$$

where $m$ is the slope of the line between inflection points of group sizes 1 and 100, c is the constant of the line and $g$ is again the number of individuals within the specified group. Based on one author's (Iu.A.G.) long experience of aerial surveys for this species and terrain, it is assumed that a herd of 100 animals was $100 \%$ detectable at the outer boundary of the transect (and beyond, up to $\sim 1.4 \mathrm{~km}$ from the aircraft; Grachev 2004), and that 1 animal was not visible at a distance of $500 \mathrm{~m}$. Hence we assume that $a=0.5, b=0.015$, $m=12.12$ and $\mathrm{c}=187.88$, giving a detectability of nearly $100 \%$ for a group of 100 animals at $1 \mathrm{~km}$ distance, and close to zero for a 'group' of 1 animal at $500 \mathrm{~m}$. It is likely that detectability at the outer boundary varies depending on conditions (Norton-Griffiths \& McConville 2007); this would increase the uncertainty in our results (McConville 2006). Together, Eqs. (2) \& (3) describe a 3D surface of detectability by distance and group size which was used to determine whether or not groups of saiga falling within the simulated survey transect were detected.

This procedure assumes that the detection probability of a group is proportional to the detection probabilities of the individuals within that group. This is simplistic as the interaction is likely to be dependent on group characteristics; closely aggregated groups may conceal individuals, while linearly spaced groups may have higher probabilities of detection for each individual.

As the group sizes were obviously being binned by observers into multiples of 10,100 or 1000 , we simulated this process by assigning each observed group a binned group size, rounded to the nearest integer of the appropriate magnitude. Hence, if a group size between 1500 and 2499 was observed in the model, it was recorded as 2000. This assumes that there is no systematic bias in the binning process with group size, which is unlikely to be true - observers may systematically over- or under-estimate the binned group size as true group size increases (Norton-Griffiths \& McConville 2007). However, a uniform distribution was used for parsimony in the absence of data on the direction of this bias.

We also carried out a sensitivity analysis to explore the interaction between population density and clumping as it affects the bias and imprecision caused by the distance-detectability relationship. A total of 1000 iterations of the simulation were carried out for a uniform group size of either 50 or 100 at 3 population densities; $1 \mathrm{~km}^{-2}$ (the observed density in 2006), 10 and $80 \mathrm{~km}^{-2}$ (the observed density in 1998). All analyses were carried out in $\mathrm{R}$ version 2.3.1 (R Development Core Team 2006).

\section{RESULTS}

The sensitivity analysis demonstrates that the presence of group sizes smaller than that at which detectability is certain biases the population estimate downward. At a group size of 50, and a $100 \%$ detectability of groups of 100 animals, the median observed population size is $76 \%$ of the true population size, whereas as expected, there is no such bias at a group size of 100 . The precision of the population estimate is dependent on density, being much lower at low densities. There is also an interaction with group size; precision is lower at larger group sizes as there is more scope for variation between runs (Fig. 2).

This analysis shows the error and bias inherent in observed population estimates when the true population size is known. In the case of the saiga datasets, we have observed population estimates and group sizes and wish to estimate the range of potential true population estimates. In Fig. 3a, we show the range of potential 'true' population sizes normalised to the observed estimate. In 1998, the observed estimate was close to the median of the 'true' population size, and the precision of this estimate was relatively high. The large group sizes had conflicting effects - they made the probability of detection, when on the transect, very high (hence the bias low), but the likelihood of the

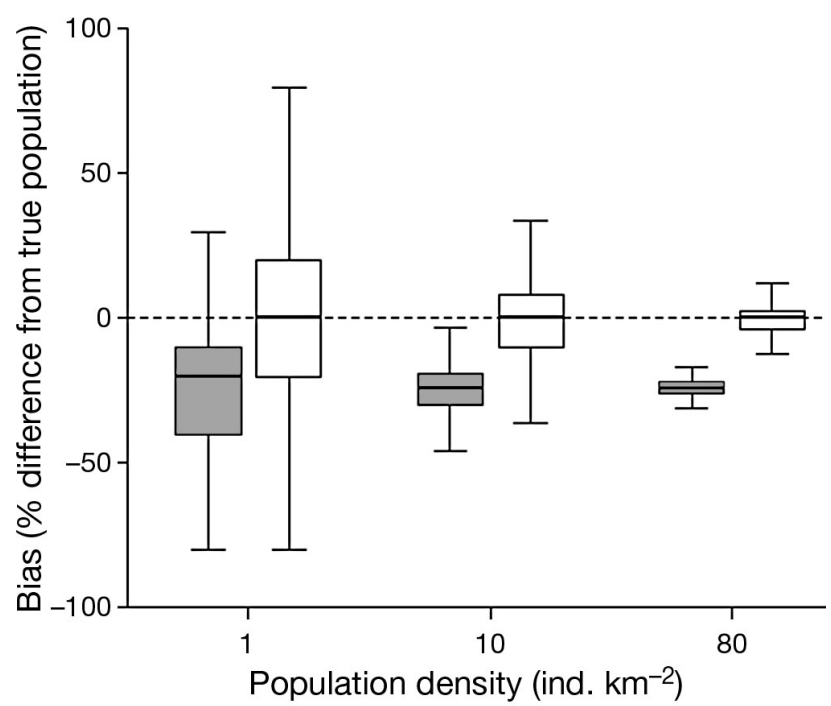

Fig. 2. Sensitivity analysis for the effect of group size and density on the precision and bias of observed population estimates when the true population size is known. Assuming a survey area of $2500 \mathrm{~km}^{-2}$, we assigned groups of size 50 (grey boxes) or 100 (white boxes) to random locations in the survey area at densities of 1,10 or 80 ind. $\mathrm{km}^{-2}$. We sampled $20 \%$ of the area $1000 \times$, with detectability dependent on distance from the centre of the $1 \mathrm{~km}$ transect. We show boxplots of the resultant distribution of observed population sizes (thick line is the median, box encloses $50 \%$ of the datapoints and whiskers are $\pm 1.5 \times$ the median) 


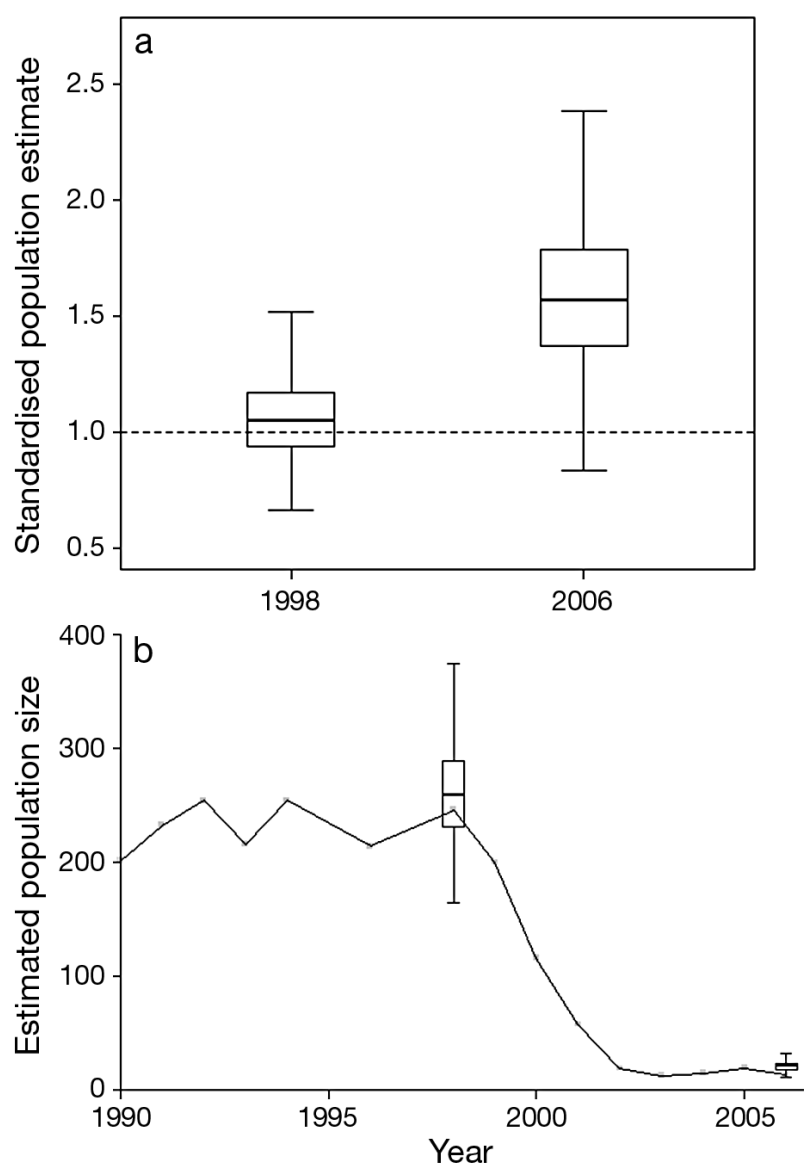

Fig. 3. (a) Box plot showing the distribution of the 'true' population estimates for 1998 and 2006, standardised to the observed population estimates (dotted line). The thick line is the median, the box encloses $50 \%$ of the datapoints and the whiskers are $\pm 1.5 \times$ the median. (b) Official data from aerial surveys of the Ustiurt saiga antelope population carried out by the Institute of Zoology, Kazakhstan, 1990 to 2006 showing the range of the likely 'true' population estimates for 1998 and 2006

group being on the transect lower (hence the precision low). However, because the density was high, the overall effect was a reasonable precision - over $50 \%$ of our repeated estimates fell within $\pm 12 \%$ of the median. In 2006, by contrast, both the population density and group size were very low. This meant that not only was the population estimate a substantial underestimate of the true population size $(63.8 \%$ of the median), but that the precision of the estimate was also much lower than in 1998. The small group size meant that detectability was very low, and the low density meant that the variation from run to run in the proportion of groups that were on the transect was high.

This error needs to be seen in the context of the overall population trend, however (Fig. 3b). Because the population collapse was so precipitate, the population in 2006 is clearly very much reduced in comparison to the situation in the late 1990s. However, the substantial error surrounding current population estimates suggests that any short-term trends in population size are unlikely to be discernible.

\section{DISCUSSION}

Although the method that we have developed for back-calculating the distribution of 'true' population sizes from observations of group sizes is crude, it is useful for disentangling the individual effects of several components of observation error. Group size has 2 conflicting effects - more clumping of the population increases the probability of detecting a group if it is on the transect, but for a given density, increases the variability between runs in whether the group is on the transect at all. There are many assumptions made in the analysis: the detection function is based on literature and expert opinion for example, and the effects of changes in survey area coverage and of biases in estimation of group size are not accounted for. Without data it is hard to speculate on the direction of these biases. Nonetheless, this exercise gives a conservative first approximation of the potential error in saiga population estimates and how this error may have changed over time.

The Government of Kazakhstan has reported consistent near-linear increases in saiga numbers in 2 of their 3 populations over the period 2003 to 2007; 77\% per annum in the Betpak-dala population and $35 \%$ per annum in the Ural population (Duisekeev \& Sklyarenko 2008). The Ustiurt population is not increasing, and this is attributed to continuing poaching in its winter range in Uzbekistan (Duisekeev \& Sklyarenko 2008). Saiga populations typically fluctuate markedly as a function of climatic conditions, particularly winter weather (Coulson et al. 2000). In recent years, a severe lack of males due to selective poaching has constrained reproductive output (Milner-Gulland et al. 2003, Fry 2004). Poaching is ongoing throughout the range of the saiga antelope (Kuhl 2008). Previous estimates of potential rates of population increase in years with favourable climatic conditions, no density dependence and no exploitation are around $30 \%$ per annum (Milner-Gulland 1994). There is some evidence that poaching pressure is currently low in parts of the Betpak-dala population's range due to low density making hunting uneconomic and to enhanced law enforcement (CMS 2006b, Kuhl 2008). A sharp reduction in livestock numbers has led to improved pasture quality (Robinson et al. 2003) and the climate has been relatively good in recent years. Hence, population growth rates of the reported magnitude are within the realms of possibility, at least in the short term. However, it 
seems likely that they are at least partly a function of changes in observation error of the kind we describe here. Our results imply that as populations recover and herd sizes increase, the degree of underestimation of population size is likely to decrease, producing an exaggerated trend of increase in apparent abundance. None of this, however, detracts from the fact that the decline in saiga antelope numbers from the late 1990s to the mid-2000s far exceeds in magnitude any effect of error and bias in abundance estimation methods; hence the validity of the listing of the saiga antelope in 2001 as Critically Endangered on the basis of a severe population decline is not in doubt.

The levels of uncertainty suggested by our analyses make it very difficult to show conclusively whether or not Kazakhstan is meeting its medium-term goal under the MOU of the CMS. On the other hand, Kazakhstan has the best survey programme of any saiga range state and has invested substantially in saiga management in recent years (Duisekeev \& Sklyarenko 2008). With relatively simple modifications to their aerial survey methods they could produce a much more robust population estimate. These include improving operational procedures and adopting a sample unit approach (NortonGriffiths 1978, Norton-Griffiths \& McConville 2007). The other range states rely on much less systematic vehicle surveys. Vehicle surveys for grassland ungulates suffer from similar bias issues to aerial surveys, with the added biases of non-random placement of transects and less consistent visibility (Harris 1996). However, several range states are planning to embark on aerial survey programmes using Kazakhstan's methods; an examination of the errors and biases inherent in the approach is therefore timely. This re-examination of monitoring methods is also highlighted as a priority action in the medium-term work programme of the CMS MOU on saiga conservation (CMS 2006a).

Our case study serves as a cautionary tale about the importance of not simply continuing with existing monitoring methods as population biology changes. Population declines often have concomitant issues of fragmentation and disturbance that change detectability, as was seen for the saiga. These changes need to be taken into account to prevent monitoring results from becoming increasingly misleading, potentially leading to incorrect conservation decision-making. We present here a simple method for retrospectively correcting existing survey data for some of these biases. This procedure is likely to be useful for other species for which potentially unreliable historical trend data are available, and for which a simple detectability model could be developed and applied retrospectively (e.g. other exploited ungulates, fish, sea turtles, birds). However, retrospective correction in the absence of detailed data on the covariates of detectability will never provide a reliable estimate of trends in abundance and the associated uncertainty, due to the large number of unquantifiable biases which remain in the data. Although lack of funds is often cited as a reason for continuing with simple, low-tech or familiar monitoring methods in order to obtain relative abundance estimates for trend detection, this may be a false economy unless variations in detectability can be properly accounted for.

Acknowledgements. This work was carried out with the financial support of INTAS (project 03-51-3579). We are also grateful for funding support from the Economic and Social Research Council (ESRC) (AK) and a Royal Society Wolfson Research Fellowship (EJMG). We thank Aline Kühl for support and advice and Marco Festa-Bianchet, Joris Cromsigt and an anonymous reviewer for useful comments. The aerial surveys were carried out with the financial support of the Government of Kazakhstan and in collaboration with Okhotzooprom. The $\mathrm{R}$ code used in the analyses is available from the authors on request.

\section{LITERATURE CITED}

Andriolo A, Piovezan U, Rodrigues Paranhos da Costa MJ, Laake J, Barbanti Duarte JM (2005) Aerial line transect survey to estimate abundance of marsh deer (Blastocerus dichotomus, Illiger, 1815). Braz Arch Biol Technol 48: 807-814

Bayliss P, Yeomans KM (1989) Correcting bias in aerial survey population estimates of feral livestock in Northern Australia using the double-count technique. J Appl Ecol 26: 925-933

Bekenov AB, Grachev IA, Milner-Gulland EJ (1998) The ecology and management of the Saiga antelope in Kazakhstan. Mammal Rev 28:1-52

Buckland ST, Anderson DR, Burnham KP, Laake JL, Borchers DL, Thomas L (2001) Introduction to distances: estimating abundance of biological populations. Oxford University Press, Oxford

Caughley G, Grice D (1982) A correction factor for counting emus from the air, and its application to counts in Western Australia. Aust Wildl Res 9:253-259

CMS (Convention on Migratory Species) (2006a) Medium term international work programme for the saiga antelope (2007-2011). CMS/SA-1/Report Annex 9. Convention on Migratory Species. www.cms.int/species/saiga/post_ session/Annex_09_MediumTerm_Int_WrkProgm_E.pdf

CMS (Convention on Migratory Species) (2006b) Revised Overview Report. CMS/SA-1/Report Annex 5. Convention on Migratory Species. www.cms.int/species/saiga/ post_session/Annex_05_Revised_Overview_Report_E.pdf

Coulson TN, Milner-Gulland EJ, Clutton-Brock T (2000) A comparison of the relative roles of density and climatic variation on fecundity rates in three contrasting ungulate species. Proc R Soc Lond B Biol Sci 267:1771-1779

Drummer TD, McDonald LL (1987) Size bias in line transect sampling. Biometrics 43:13-21

Duisekeev BZ, Sklyarenko SL (2008) Conservation of saiga antelopes in Kazakhstan. Saiga News Issue 7. www.saigaconservation.com/saiga_news.html

Fry M (2004) The status of the Saiga antelope in the Ustiurt region of western Kazakhstan. MSc thesis, Imperial College London 
Grachev IuA (2004) Saiga. In: Metodi uchota osnovnikh okhotniche-promislobikh I redkikh vidov zhivotnikh Kazakhstana (Fundamental methods of assessment for hunted and rare species of animals in Kazakhstan). Institute of Zoology, Almaty

Gregory RD, Gibbons DW, Donald PF (2004) Bird census \& survey techniques. In: Sutherland WJ, Newton I, Green RE (eds) Bird ecology \& conservation; a handbook of techniques. Oxford University Press, Oxford, p 17-56

Guenzel RJ (1997) Estimating pronghorn abundance using aerial line transect sampling. Wyoming Game and Fish Department, Cheyenne. www.ruwpa.st-and.ac.uk/ distance.book/pronghorn.html

Harris R (1996) Wild ungulate surveys in grassland habitats: satisfying methodological assumptions. Chin J Zool 31: 16-21. http://people.montana.com/ rharris/Satisfying \% 20Methodological\%20Assumptions.doc

Hochachka WM, Fiedler W (2008) Trends in trappability and stop-over duration can confound interpretations of population trajectories from long-term migration ringing studies. J Ornithol 149:375-391

Jolly GM (1969) Sampling methods for aerial censuses of wildlife populations. East Afr Agric For J 34(Spec Issue):46-49

Kuhl A (2008) Conservation ecology of the saiga antelope. $\mathrm{PhD}$ thesis, Imperial College, London

Lorenzen K, Almeida O, Arthur R, Garaway C, Nguyen Khoa S (2006) Aggregated yield and fishing effort in multispecies fisheries: an empirical analysis. Can J Fish Aquat Sci 63:1334-1343

McConville AJ (2006) Modelling the biases in aerial survey techniques of the saiga antelope (Saiga tatarica) in Kazakhstan. MSc thesis, Imperial College London

Milner-Gulland EJ (1994) A population model for the management of the saiga antelope. J Appl Ecol 31: 25-39

Milner-Gulland EJ, Kholodova MV, Bekenov A, Bukreeva OM, Grachev IA, Amgalan L, Lushchekina AA (2001) Dramatic declines in saiga antelope populations. Oryx 35: 340-345

Editorial responsibility: Wayne Linklater, Wellington, New Zealand
Milner-Gulland EJ, Bukreeva OM, Coulson TN, Lushchekina AA, Kholodova MV, Bekenov AB, Grachev IuA (2003) Reproductive collapse in saiga antelope harems. Nature 422:135

Norton-Griffiths M (1978) Counting animals, 2nd edn. African Wildlife Foundation, Nairobi

Norton-Griffiths M, McConville AJ (2007) Technical evaluation of the aerial survey programme for saiga antelope (Saiga tatarica) with recommendations for improvements. Final Report. ZGF-Project No. 1293/02 Saiga Schutz Kasachstan/Evaluation der Tierzahlungen. Frankfurt Zoological Society, Frankfurt

> Norvell RE, Howe FP, Parrish JR (2003) A seven-year comparision of relative abundance and distance-sampling methods. Auk 120:1013-1028

> Pollock KH, Nichols JD, Simons TR, Farnsworth GL, Bailey LL, Sauer JR (2002) Large scale wildlife monitoring studies: statistical methods for design and analysis. Environmetrics 13:105-119

R Development Core Team (2006) R: A Language and Environment for Statistical Computing. R Foundation for Statistical Computing, Vienna, Austria. www.R-project.org

Robinson S, Milner-Gulland EJ, Alimaev I (2003) Rangeland degradation in Kazakhstan during the Soviet era: reexamining the evidence. J Arid Environ 53:419-439

Samuel MD, Pollock KH (1981) Correction of visibility bias in aerial surveys where animals occur in groups. J Wildl Manag 45:993-997

Samuel MD, Garton EO, Schlegel MW, Garson RG (1987) Visibility bias during aerial surveys of elk in Northcentral Idaho. J Wildl Manag 51:622-630

Schauster ER, Gese EM, Kitchen AM (2002) An evaluation of survey methods for monitoring swift fox abundance. Wildl Soc Bull 30:464-477

Stephens PA, Sutherland WJ (1999) Consequences of the Allee effect for behaviour, ecology and conservation. Trends Ecol Evol 14:401-405

- Walters C (2003) Folly \& fantasy in the analysis of spatial catch rate data. Can J Fish Aquat Sci 60:1433-1439

Submitted: July 29, 2008; Accepted: October 24, 2008

Proofs received from author(s): January 8, 2009 